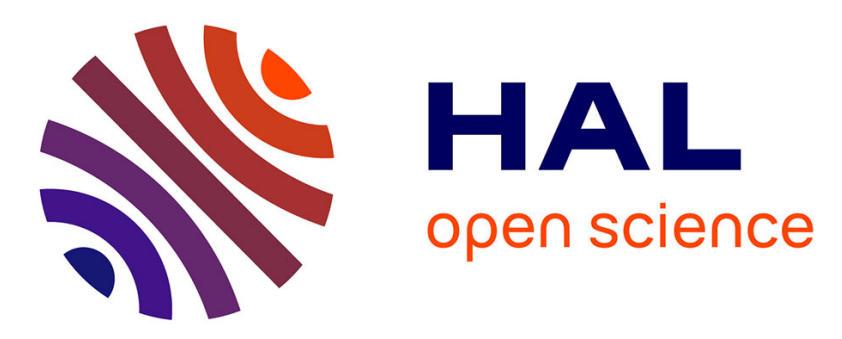

\title{
Coherent beam combining of two femtosecond fiber chirped-pulse amplifiers
}

Louis Daniault, Marc Hanna, Laurent Lombard, Yoann Zaouter, Eric Mottay, Didier Goular, Pierre Bourdon, Frédéric Druon, Patrick Georges

\section{- To cite this version:}

Louis Daniault, Marc Hanna, Laurent Lombard, Yoann Zaouter, Eric Mottay, et al.. Coherent beam combining of two femtosecond fiber chirped-pulse amplifiers. Optics Letters, 2011, 36 (5), pp.621. hal-00609851

\section{HAL Id: hal-00609851 \\ https://hal.science/hal-00609851}

Submitted on 20 Jul 2011

HAL is a multi-disciplinary open access archive for the deposit and dissemination of scientific research documents, whether they are published or not. The documents may come from teaching and research institutions in France or abroad, or from public or private research centers.
L'archive ouverte pluridisciplinaire HAL, est destinée au dépôt et à la diffusion de documents scientifiques de niveau recherche, publiés ou non, émanant des établissements d'enseignement et de recherche français ou étrangers, des laboratoires publics ou privés. 


\title{
Coherent beam combining of two femtosecond fiber chirped-pulse amplifiers
}

\author{
L. Daniault, ${ }^{1, *}$ M. Hanna, ${ }^{1}$ L. Lombard,${ }^{2}$ Y. Zaouter, ${ }^{3}$ E. Mottay,${ }^{3}$ \\ D. Goular, ${ }^{2}$ P. Bourdon, ${ }^{2}$ F. Druon, ${ }^{1}$ and P. Georges ${ }^{1}$ \\ ${ }^{1}$ Laboratoire Charles Fabry de l'Institut d'Optique, UMR 8501 CNRS, Université Paris Sud, 91127 Palaiseau, France \\ ${ }^{2}$ Office National d'Etudes et de Recherches Aérospatiales, Département d'Optique Théorique et Appliquée, \\ Chemin de la Hunière, 91761 Palaiseau, France \\ ${ }^{3}$ Amplitude Systèmes, 6 allée du Doyen Georges Brus, 33600 Pessac, France \\ ${ }^{*}$ Corresponding author: louis.daniault@institutoptique.fr
}

Received December 7, 2010; revised January 19, 2011; accepted January 21, 2011;

posted February 1, 2011 (Doc. ID 139352); published February 18, 2011

We demonstrate coherent beam combining of two femtosecond fiber chirped-pulse amplifiers seeded by a common oscillator. Using a feedback loop based on an electro-optic phase modulator, an average power of $7.2 \mathrm{~W}$ before compression is obtained with a combining efficiency of $90 \%$. The spatial and temporal qualities of the oscillator are retained, with a recombined pulse width of $325 \mathrm{fs}$. This experiment opens up a way to scale the peak/average power of ultrafast fiber sources. (c) 2011 Optical Society of America

OCIS codes: $\quad 060.2320,140.3298,140.7090$.

Ytterbium-doped fiber amplifiers present great advantages in femtosecond regime, including high optical-tooptical efficiency, good thermal handling and high spatial beam quality. However, the tight confinement of the beam inside the fiber and the long interaction length can lead to strong nonlinear effects at high energies, degrading the temporal profile of the output pulses. This constitutes a limit to femtosecond fiber chirped-pulse amplifier (CPA) setups.

A widely investigated way to reduce nonlinearities consists in enlarging the mode field area of the fiber amplifier, so that nonlinear effects appear at higher energies $[1,2]$. However, the core scaling capability is limited by the transverse multimode character that inevitably appears for large core fibers, reducing the beam quality of the laser source. Moreover, the onset of self-focusing at peak powers of the order of $4 \mathrm{MW}$ is independent of the core size and represents a hard limit for the peak power that can be handled in silica fibers.

Another way to scale the energy is to use several amplifiers and coherently combine their outputs in free space to get the sum of the power while maintaining the beam quality and avoiding detrimental effects at high peak powers in the fiber. This technique is already used in CW [3] and studied in nanosecond regimes [4], and is scalable in terms of achievable energy.

In another context, coherent addition of femtosecond optical pulses has been observed recently with two pulses without any spectral overlap, allowing the generation of single cycle pulses [5]. In this case, the motivation is not to scale the power but to obtain the shortest possible pulse.

Here we report on the coherent beam combining of two identical femtosecond Yb-doped fiber CPAs, using active control of the relative phase between the amplifiers by use of an integrated electro-optics phase modulator. Two $4 \mathrm{~W}$ femtosecond amplifiers are combined, and the output power is $7.2 \mathrm{~W}$, resulting in a combining efficiency of $90 \%$. The spatial, temporal, and spectral characteristics of a single amplifier are completely recovered in the combined beam, with output pulse duration of
325 fs. This combining experiment opens a novel way for energy/average power scaling of femtosecond amplifiers.

In $\mathrm{CW}$ coherent combining experiments, the relative phase between the beams is measured and controlled through a feedback loop. In femtosecond regime, since the optical bandwidth is larger, the optical phase must be matched over the whole bandwidth to efficiently combine the pulses. In other words, the phase, group delay, group-delay dispersion, and so on, should be matched. The tolerance of the system to mismatch of these parameters is inversely related to the pulse width. In what follows, i.e., for pulse durations above $100 \mathrm{fs}$, we show that a static adjustment of the group delay and groupvelocity dispersion, along with an active correction of the phase, is sufficient to efficiently combine the pulses. Indeed, the group delay and group-delay dispersion fluctuations between the pulses are sufficiently weak so that the final pulse width is not affected.

Our experimental setup, depicted in Fig. 1, begins with a femtosecond air-cooled bulk $\mathrm{Yb}^{3+}: \mathrm{KYW}$ oscillator delivering $260 \mathrm{fs}$ pulses at $1030 \mathrm{~nm}$ and $35 \mathrm{MHz}$ repetition rate with $1 \mathrm{~W}$ average power. It is followed by a stretcher that broadens the pulses to $150 \mathrm{ps}$. A half-wave plate with a polarizing beam splitter allows us to separate the seed into two arms with adjustable input power for each. The first one consists of a fiber-coupled $\mathrm{LiNbO}_{3}$ integrated phase modulator followed by a $1.2 \mathrm{~m} \mathrm{Yb}$-doped doubleclad polarization-maintaining $30 / 150 \mu \mathrm{m}$ large mode area (LMA) fiber amplifier. The second one contains a $2.40 \mathrm{~m}$ single-mode fiber, a free-space delay line, and another $1.2 \mathrm{~m} \mathrm{Yb}$-doped double-clad 30/150 $\mu \mathrm{m}$ LMA fiber amplifier. The aim of the single-mode fiber is to match the group-velocity dispersion of both arms, and it also acts as a coarse delay matching element. Both amplifiers are seeded with $100 \mathrm{~mW}$ of average power and are pumped to deliver identical powers. At this repetition rate, we operate in completely linear regime, so that self-phase modulation-induced degradation of the combining efficiency does not take place [6]. The outputs of the fiber amplifiers are collimated and overlapped on a 50/50 beam splitter. Provided that the pulses have the same phase, 




Fig. 1. (Color online) Experimental setup of the femtosecond coherent combining experiment.

arrival time, temporal shape, polarization, and spatial profile, they can be recombined coherently. The constructive output of the beam splitter goes to the compressor that exhibits a $60 \%$ efficiency. The other one is followed by a photodiode with a bandwidth of $1 \mathrm{MHz}$ that detects a signal to be minimized.

The relative phase between the pulses is controlled using the technique described in Ref. [3]. A small voltage modulation at $250 \mathrm{kHz}$ is added to the control signal that drives the phase modulator, yielding a small modulation $(0.1 \mathrm{rad})$ of the relative phase between the two arms. At the output of the photodiode, a lock-in amplifier allows the detection of the small induced amplitude modulation. The resulting error signal is directly proportional to the optical phase difference between the arms. A digital controller then maintains the phase difference close to 0 by adding an offset voltage to the small modulation voltage and manages $2 \pi$ phase jumps to stay in the phase modulator input voltage range. Since the modulator bandwidth is $>1 \mathrm{GHz}$, the phase jump is instantaneous for pulses at $35 \mathrm{MHz}$ repetition rate. The integration time of the lock-in amplifier is set to $20 \mu \mathrm{s}$, meaning that the maximum phase noise frequency that can be corrected is $25 \mathrm{kHz}$. The controller parameters are set to minimize the RMS phase fluctuations. The overall feedback loop bandwidth in our case is around $1 \mathrm{kHz}$, determined by the controller integration. However, this method was shown to be compatible with larger bandwidths, as demonstrated in Ref. [7].

The photodiode signal versus time is shown in Fig. 2 , both in free-running and locked phase regimes, over $100 \mathrm{~s}$. The free-running signal evolves arbitrarily between the in-phase and out-of-phase states, which determines a contrast $\left(I_{\max }-I_{\min }\right) /\left(I_{\max }+I_{\min }\right)$ of $84 \%$. This contrast is very sensitive to the spectral intensity of each pulse and depends more loosely on the group delay and group-delay dispersion discrepancies. In phase-locked operation, the signal shows residual intensity fluctua-

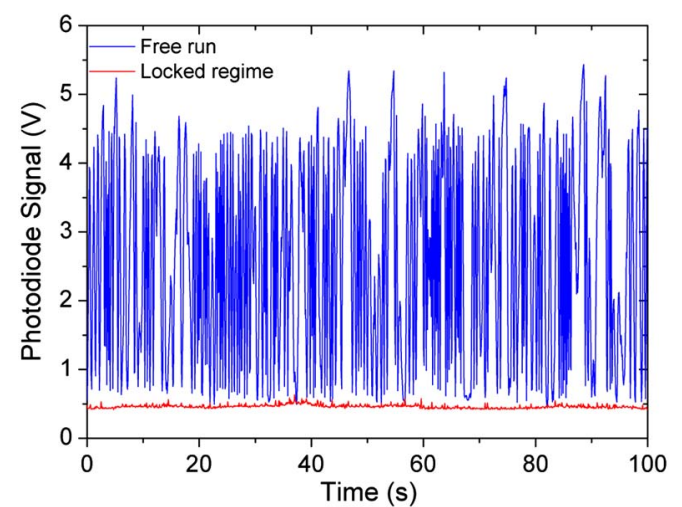

Fig. 2. (Color online) Photodiode signal versus time, over $100 \mathrm{~s}$, in free run and phase-locked regime.

tions that correspond to an RMS phase noise of $\lambda / 40$ over the period of $100 \mathrm{~s}$, assuming that the intensity noise is negligible. No readjustment of the delay line is necessary to keep the system locked over hours, indicating that the group-delay drift is small compared to the pulse duration.

Figure 3 shows the phase noise spectral density at the output of the recombination system, computed from the intensity measurement, for free-running and phaselocked operation. Most of the phase noise content is below $1 \mathrm{kHz}$, and our setup is therefore well adapted to correct this phase fluctuation bandwidth. In particular, the acoustic fluctuations above $100 \mathrm{~Hz}$ are compensated by the phase-locking electronics.

Each amplifier delivers $4 \mathrm{~W}$ with a $10 \mathrm{~W}$ pump power. The recombined power is measured to be $7.2 \mathrm{~W}$ before compression, which corresponds to a combining efficiency $P_{\text {out }} /\left(P_{1}+P_{2}\right)$ of $90 \%$ and a compressed power of $4.3 \mathrm{~W}$. The output polarization state of each amplifier is measured to be linear to more than $99 \%$. Therefore, polarization has little effect on the efficiency. To achieve this efficiency, the spatial overlap of the beams at the output of the beam splitter must be very carefully optimized. Any difference between the wavefronts, such as defocus and tilt, or in the beam profiles, such as ellipticity and beam position, results in a drastically reduced combining efficiency. The intensity profiles of each arm separately and of the combined beam are shown in Fig. 4. The individual profiles do not exhibit exactly the same shape, and the combined beam is intermediate. This slight



Fig. 3. (Color online) Phase noise density of the recombination, in free run (black) and locked regime (red). 




Fig. 4. (Color online) Intensity profiles of (left), (center) the individual channel beams and of (right) the recombined beam at the output of the compressor.

spatial discrepancy contributes to the nonperfect combining efficiency.

Finally, we focus on the temporal and spectral properties of the pulses, for each channel independently and for the recombined output. The autocorrelations and spectra are plotted in Fig. 5. The autocorrelations exhibit a very good match with the sech ${ }^{2}$ temporal shape, so that a 1.54 deconvolution factor is used to infer the pulse widths. The pulse durations for each isolated arm are $315 \mathrm{fs}$ for the first one and $335 \mathrm{fs}$ for the second one. Thus, for the same compression, the pulses do not have exactly the same duration, indicating a small group-delay dispersion discrepancy. The recombined pulse exhibits 325 fs duration, corresponding to the mean of the initial durations. However, a simple model can be used to show that the contribution of this temporal mismatch to the overall combining efficiency is negligible compared to spatial aspects.

The recombined spectrum is also very similar to the isolated ones. Again, the recombined spectrum is between both individual spectra, with a $4.3 \mathrm{~nm}$ FWHM. The time-bandwidth product of the recombined pulse is 0.39 , close to the Fourier-transform-limited product of 0.315 for a sech ${ }^{2}$ profile. This slightly degraded time-bandwidth product is the same for a single amplifier and is due to a nonperfect alignment of the stretcher and compressor, resulting in high-order spectral phase mismatch. The temporal quality after the combining process is therefore very well preserved.

To conclude, we demonstrate coherent beam combining of two femtosecond fiber CPAs using a single stretcher and compressor, resulting in an output power of $7.2 \mathrm{~W}$ before compression, with $90 \%$ efficiency. The RMS residual phase error is $\lambda / 40$, and the final pulse width is $325 \mathrm{fs}$, with a good spatial and temporal quality. Theoretical considerations to evaluate the tolerance of the system to residual discrepancies between the arms in terms of phase, group delay, dispersion, and $B$ integral indicate that these systems are realistically scalable. To our knowledge, only one similar experiment has been done independently by another research team and reported very recently, using an alternative method based on polarization for the phase control [8] and for longer pulses. However, the use of the polarization state to generate the error signal implies that only two amplifiers can be combined by one system, and the scalability of this scheme might be challenging. In our case, although the results were obtained with two amplifiers using a beam splitter, the phase feedback loop is compatible with scalable combining geometries such as the tiled aperture technique. Considering that a seed of $10 \mathrm{~mW}$ is sufficient to overcome amplified spontaneous emission in our fibers, this setup is thus already applicable to a few tens of amplifiers. Higher scalability can be obtained with preampli-
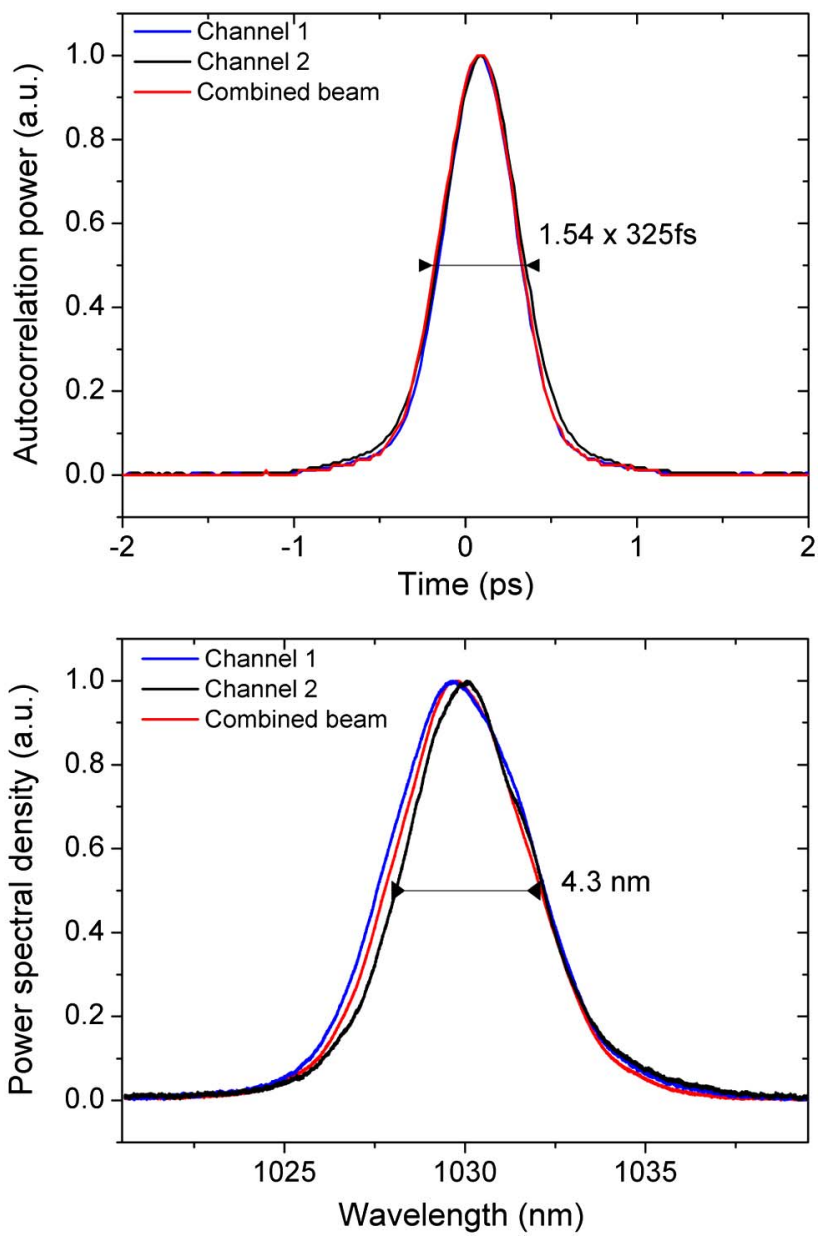

Fig. 5. (Color online) (top) Autocorrelation traces and (bottom) optical spectra for each channel independently (blue and black) and for the recombined beam (red).

fiers that might be implemented in an all-fiber setup. These experiments represent a promising way towards future high-average and peak power laser systems.

The research described here has been supported by Triangle de la Physique contract 2009-026T.

\section{References}

1. Y. Zaouter, J. Boullet, E. Mottay, and E. Cormier, Opt. Lett. 33, 1527 (2008).

2. F. Röser, T. Eidam, J. Rothhardt, O. Schmidt, D. N. Schimpf, J. Limpert, and A. Tünnermann, Opt. Lett. 32, 3495 (2007).

3. T. Shay, V. Benham, J. T. Baker, A. D. Sanchez, D. Pilkington, and C. A. Lu, IEEE J. Sel. Top. Quantum Electron. 13, 480 (2007).

4. E. C. Cheung, M. Weber, and R. R. Rice, in Advanced SolidState Photonics, OSA Technical Digest Series (CD) (Optical Society of America, 2008), paper WA2.

5. G. Krauss, S. Lohss, T. Hanke, A. Sell, S. Eggert, R. Huber, and A. Leitenstorfer, Nat. Photon. 4, 33 (2010).

6. S. Jiang, M. Hanna, F. Druon, and P. Georges, Opt. Lett. 35, 1293 (2010).

7. T. M. Shay, J. T. Baker, A. D. Sancheza, C. A. Robin, L. C. L. Vergien, A. Flores, C. Zerinque, D. Gallant, C. A. Lu, B. Pulford, T. J. Bronder, and A. Lucero, in Advanced Solid-State Photonics, OSA Technical Digest Series (CD) (Optical Society of America, 2010), paper AMA1.

8. E. Seise, A. Klenke, J. Limpert, and A. Tünnermann, Opt. Express 18, 27827 (2010). 